The prevalence of EEG abnormalities in AS is $80 \%$, and 3 main patterns are present, independent of epileptic seizures: persistent rhythmic $4-6 \mathrm{~Hz}$ activity, anterior delta activity of 2-3 Hz with superimposed spikes and sharp waves, and posterior 3-4 Hz high amplitude waves with spikes and sharp waves, especially on eye closure. AS patients with a deletion of chromosome 15q11-13 have more prominent EEG abnormalities than those with other genetic disorders of chromosome 15 region. EEG abnormalities are helpful in the diagnosis of AS at an early age. (Laan LAEM, Vein AA. Brain Dev 2005;27:80-87).

The severity of developmental disturbance in AS is not invariably related to the severity of epilepsy, although repetitive nonconvulsive status epilepticus can sometimes result in transient or permanent mental and motor deterioration. (Ohtsuka Y et al. Brain Dev 2005;27:95-100).

\title{
NONFASTING VERSUS INITIAL FASTING KETOGENIC DIET
}

A retrospective evaluation of the ketogenic diet $(\mathrm{KD})$ was conducted comparing efficacy and tolerability of the diet with or without initial fasting and fluid restriction and involving university centers in Seoul, Korea. Of 41 children with intractable epilepsy treated with the nonfasting diet (NFKD), 14 (34.1\%) were seizure-free for at least 3 months, and in 83 receiving the initial fasting diet (IFKD), 29 (34.9\%) were seizure free. The patients' mean age was $4.1(+/-1.5)$ years in the NFKD group and $5.3(+/-1.6)$ years in the IFKD group. The incidence of various seizure types was not significantly different in the 2 groups. Strong urinary ketosis developed within $2.4+/-1.2$ days in the NFKD group, compared to $1.9+/-$ 1.3 days in the IFKD group (not significant, $\mathrm{P}>0.05$ ). Among early complications, dehydration was significantly less frequent in the NFKD group (12.2\%) than in the IFKD group $(62.7 \% ; \mathrm{P}<0.05)$. The incidence of other complications was not significantly different in the NF and IF groups, including hypoglycemia (4.9 v 4.8\%), nausea and vomiting ( $36 \mathrm{v}$

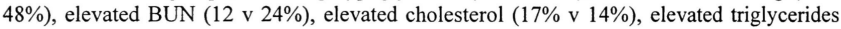
(36 v 36\%). Patients in the NFKD group were hospitalized for a mean period of $5.8(+/-2.7)$ days and those in the IFKD group for $7.8(+/-1.7)$ days $(\mathrm{P}<0.05)$. (Kim DW, Kang HC, Park JC, Kim HD. Benefits of the nonfasting ketogenic diet compared with the initial fasting ketogenic diet. Pediatrics Dec 2004;114:1627-1630). (Reprints: HD Kim MDPhD, Department of Pediatrics, Severance Hospital, Yonsei University College of Medicine, 134, Shinchon-dong, Seodaemun-gu, Seoul 120-752, Korea).

C OMMENT. The nonfasting ketogenic diet, in the above retrospective study, was of equal efficacy, and its introduction was better tolerated than the initial fasting method. The $\mathrm{KD}$ was first introduced for the treatment of epilepsy at the Mayo Clinic (Wilder RM. Mayo Clin Bull 1921;2:307). In the Mayo Clinic method, unlike the later Johns Hopkins protocol (Livingston S. Postgrad Med 1951;10:333-336), the $\mathrm{KD}$ is introduced without initial fasting and usually, without admission to hospital. The ratio of ketogenic to antiketogenic (K:AK) items in the diet is reversed gradually, starting at a ratio of 1:1.1 on day 1 and increasing to $2.8: 1$ by the $4^{\text {th }}$ day, or until ketones are found in the urine. In young children, a ratio of $3 ; 1$ is usually sufficient to maintain ketosis, and generally the higher $4: 1$ ratio is necessary only in older children. In my experience with the diet, beginning at the Mayo Clinic in the early 1960s and continuing at Children's Memorial Hospital in Chicago, the NFKD method has 
proven satisfactory and free from serious side effects. (Millichap JG. Nutrition, Diet, and Your Child's Behavior. Springfield, IL; Charles C Thomas, 1986; Millichap et al. Epilepsia 1964;5:239-255; Am J Dis Child 1964;107:593-604; JAMA 1966;198:210).

A flurry of papers on the ketogenic diet has appeared in recent months. A retrospective study has shown that the $\mathrm{KD}$ can be successfully initiated on an outpatient basis without starvation, fluid or caloric restriction (Vaisleib II et al. Pediatr Neurol 2004;31:198202). The worldwide use of the $\mathrm{KD}$ has been established by communicating with 73 academic centers in 41 countries using the Internet. The average number of patients enrolled was 72 per country, with 5 new patients annually. Common difficulties included failure to avoid rice intake, intolerance symptoms with the higher $\mathrm{K}: \mathrm{AK}$ 4:1 ratio, using the Hopkins protocol. A website is now available (Kossoff EH, McGrogan JR. Epilepsia 2005;46:280289).

In a Korean multicenter study involving 199 patients (87 using the IFKD Hopkins protocol and 112 the NFKD), 66 (34\%) successfully completed or maintained the diet. By modifying the protocol to omit the fasting period, especially in young children, acute dehydration was prevented, with no difference in the time to ketosis or in the efficacy of the diet. Five patients died related to lipoid aspiration pneumonia, serious infectious disease, and nutritional problems (Kang HC et al. Epilepsia 2005;46:272-279). The ketogenic diet was successful using gastrostomy tube feeding to ensure compliance, in the treatment of 12 children with static encephalopathy and intractable seizures (Hosain SA et al. Pediatr Neurol 2005;32:81-83).

\section{LEARNING DISABILITIES}

\section{NEURAL BASIS FOR DYSLEXIA AND PICTURE NAMING DEFICITS}

Eight dyslexic subjects, aged $20+/-0.9$ years, university students who were impaired on measures of reading, spelling and naming speed, matched for age and general ability with 10 control subjects, were scanned using PET while reading words and naming pictures, in a study at the Institute of Psychiatry, University College, and the Institute of Neurology, London, UK. Dyslexic subjects showed reduced activation in a left occipitotemporal area during both word reading and picture naming. The findings point to a common neurological basis for deficits in word reading and picture naming in developmentâl dysiexia. (MúcCrưiy EJ, Mechelli A, Frith U, Price CJ. More than words: a common neural basis for reading and naming deficits in developmental dyslexia? Brain February 2005;128:261-267). (Respond: Eamon J McCrory, Insatitute of Psychiatry, Department of Psychology, De Crespigny Park, London SE5 8AF, UK).

COMMENT. Previous studies have demonstrated abnormal activation of the left occipitotemporal area during word processing in dyslexia (Salmelin R et al. Ann Neurol 1996;40:157-162; Shaywitz BA et al. Biol Psychiatry 2002;52:101-110). Children learn to name pictures of objects before they can read. The above UK study shows that an area of the brain involved in word reading is also activated in picture naming. The authors propose that the diagnosis and treatment of a delay in picture naming skills in preschool children may influence the later acquisition of reading performance. 